\title{
Three Notions of Dynamicness in Language*
}

\author{
Daniel Rothschild ${ }^{\dagger} \quad$ Seth Yalcin ${ }^{\ddagger}$ \\ d.rothschild@ucl.ac.uk yalcin@berkeley.edu
}

April 8, 2016

\begin{abstract}
We distinguish three ways that a theory of linguistic meaning and communication might be considered dynamic in character. We provide some examples of systems which are dynamic in some of these senses but not others. We suggest that separating these notions can help to clarify what is at issue in particular debates about dynamic versus static approaches within natural language semantics and pragmatics.
\end{abstract}

In the seventies and early eighties, theorists like Karttunen, Stalnaker, Lewis, Kamp, and Heim began to 'formalize pragmatics', in the process making the whole conversation or discourse itself the object of systematic formal investigation (Karttunen [1969, 1974]; Stalnaker [1974, 1978]; Lewis [1979]; Kamp [1981]; Heim [1982]; see also Hamblin [1971], Gazdar [1979]). This development sometimes gets called "the dynamic turn". Much of this work was motivated by a desire to model linguistic phenomena that seemed to involve a special sensitivity to the preceding discourse (with presupposition and anaphora looming especially large) - what we could loosely call dynamic phenomena. The work of Heim [1982] in particular showed the possibility of a theory of meaning which identified the meaning of a sentence with (not truth-conditions but) its potential to influence the state of the conversation. The advent of this kind of dynamic compositional semantics opened up a new question at the semantics-pragmatics interface: which seemingly dynamic phenomena are best handled within the compositional semantics (as in a dynamic semantics), and which are better modeled by appeal to a formal 'dynamic pragmatics' understood as separable from, but perhaps interacting with, the compositional semantics? Versions of this question continue to be debated within core areas of semantic-pragmatic inquiry - for a small sampling see, for instance, recent work on presupposition projection (Beaver [2001, 2008], Schlenker [2007, 2008, 2009], Rothschild [2011],

\footnotetext{
*Thanks to Jim Pryor and Lawerence Valby for essential conversations.

${ }^{\dagger}$ Department of Philosophy, University College London, Gower Street, London WC1E 6BT UK

${ }^{\ddagger} 134$ Moses Hall, Department of Philosophy, University of California, Berkeley, CA 94720 USA
} 
Beaver and Geurts [2014], Stalnaker [2014]), on counterfactuals (von Fintel [2001], Veltman [2005], Gillies [2007], Moss [2012]) and on epistemic modals and indicative conditionals (Veltman [1996], Aloni [2001], Gillies [2004], Yalcin [2007, 2012c, 2015], Kolodny and MacFarlane [2010], Willer [2012, 2013a,b], Bledin [2014], Stalnaker [2014]). This issue forms a key aspect of the large-scale question what kind of shape a theory of meaning for natural language should take. $^{1}$

This paper is not an intervention into any specific debate at the semanticspragmatics boundary. Rather, we want to do some conceptual ground-clearing. Several notions of "dynamicness" are often in play in such debates. Our aim is to isolate some of these concepts and sharpen them. We will suggest that separating these notions can help to clarify what is at issue in particular debates about dynamic versus static approaches within natural language semantics and pragmatics. Certain considerations may favor some notions of dynamicness but not others. The presence of one kind of dynamicness might be motivated independently of the presence of other kinds of dynamicness.

We isolate three ways that a fragment of language might be considered dynamic, corresponding to three different notions of dynamicness. To a rough first approximation, these are:

Compositional dynamicness. The compositional semantic values of sentences are context-change potentials-functions which map a conversational state to a new conversational state.

Conversation systems dynamicness. The context-change potentials of the sentences of the language are not each equivalent to an operation which adds a proposition to the background information of the conversation.

Discourse dynamicness. The truth-conditions associated with a discourse as a whole cannot always be understood as the result of first associating the sentential parts of the discourse with truth-conditions and then combining these truth-conditions.

We refine and discuss each notion in its own section below. We will provide some examples of systems which are dynamic in some of these senses but not others. Before that, we review some terminology and some background assumptions.

\footnotetext{
${ }^{1}$ Recent discussions here include von Fintel and Gillies [2007], Schlenker [2009], Rothschild [2011], Lewis [2012, 2014], Dever [2013], Stalnaker [2014], Rothschild and Yalcin [2015].
} 


\section{Preliminaries}

Following many approaches in semantic and pragmatics, we take it that it is theoretically fruitful to work with some formal notion of a conversational state, in part so that we can formally model speech acts from the perspective of their characteristic effects on the state of the conversation in which they occur. Different frameworks model states of conversations with different sorts of objects. Stalnaker [1978], for instance, models the state of a conversation as a set of possible worlds, the set of worlds compatible with the presuppositions of the interlocutors (a context set in his terminology). Lewis [1979] models conversational states via an abstract 'conversational scoreboard' including a number of dimensions beyond a context set, among them a ranking of comparative salience of objects, a parameter tracking the prevailing standards of precision, and a component representing the possible plans of the interlocutors. Kamp [1981] models the state of a conversation with a discourse representation structure, a certain kind of syntactic object. Heim [1982] models the state of a conversation as a file, which formally amounts to a pair of a set of variables together with a set of world-variable assignment pairs. Roberts [1996, 2012] models a conversational state as a tuple of different kinds of information, one of which is a 'question-under-discussion stack' used to model relevance and the inquiries directing conversation. Veltman [1996] models conversational states as sets of worlds paired with a preorder over worlds, the latter intended to model expectation or normality. Willer [2013a] models conversational states as sets of sets of worlds, using these to (inter alia) formally capture what it is for a possibility to be live in conversation. Yalcin [2012b] models conversational states as sets of probability spaces, using these to deal with probability operators and conditionals. And so on. ${ }^{2}$ We will speak throughout of a domain of conversational states, but we will be neutral as to the question how to model these states. ${ }^{3}$

We take it that when one performs a speech act, one (inter alia) makes a bid to induce a certain dynamical change to the state of the conversation, a change that has something intimately to do with the meaning (compositional semantic value) of the linguistic expression that is uttered. We take this simple idea to

\footnotetext{
${ }^{2}$ And on. For just a few more examples of the diverse ways in which states of conversation have been modeled, see Groenendijk [1999], Aloni [2001], Groenendijk et al. [1996], Bittner [2011], Dekker [2012], Murray [2014].

${ }^{3}$ Is a (nondefective) conversation always adequately representable using single conversational state, or should we allow for multiple, perhaps different conversational states, one for each agent in the discourse? We tend to think of matters in former way, broadly in the style of Stalnaker [2002]; thus we will often speak of the conversational state at a given point in a conversation. But nothing we say will turn on this, and officially we are neutral. See Stalnaker [2014] for one recent relevant discussion.
} 
be common ground between dynamic and non-dynamic theorists.

Relatedly, we take it that every sentence (or sentence in context ${ }^{4}$ ) has a context-change potential (CCP). This is a function which, roughly speaking, captures the change that uttering the sentence produces in the state of the conversation when the speech act is accepted. A context-change potential is a function from conversational states to conversational states.

We say "roughly speaking" because, as emphasized by Stalnaker in a number of places (Stalnaker [1978], Stalnaker [1998], Stalnaker [2014]), uttering a sentence always produces changes to the state of the conversation which are not themselves part of the context-change potential associated with the sentence. For example, if one says 'It's raining in Brooklyn' in the context of a normal conversation, the associated conversational state will presumably be immediately updated with the information that one has just asserted something. This change to the conversational state owes, not to the CCP of 'It's raining in Brooklyn', but rather to the truism that facts that become mutually evident to interlocutors in conversation normally become mutually presupposed in the conversation, and thus are added to the background information incorporated in the conversational state. In the normal case, the CCP associated with an utterance of a sentence $\phi$ will act on a conversational state which already includes the information that $\phi$ was just uttered. A textbook idea for the $\mathrm{CCP}$ of the sentence 'It's raining in Brooklyn' would be that it is a function that maps an arbitrary conversational state $c$ to a conversational state that captures all the information that was already part of $c$, plus the information that it's raining in Brooklyn. The CCP of a sentence is always of a function of what the sentence means.

\section{Compositional dynamicness}

This brings us to our first notion of dynamicness. Although the term contextchange potential emerged in the dynamic semantics literature, it is important to be clear that one can speak with propriety of the context change potential of a sentence without assuming in advance that the sentence, or the language it is part of, requires a dynamic compositional semantics. The term as we use it is neutral on that issue. Those who, like Stalnaker [2014], embrace conventional

\footnotetext{
${ }^{4} \mathrm{By}$ 'context' in 'sentence in context', we mean the sense in play in, for example, Kaplan [1977/1989]: in this sense, the context is the concrete location where the discourse takes place, something formalizable as a centered world. The 'context' in 'context-change potential' adverts, by contrast, to a different notion of context-namely, the notion of a conversational state. For some discussion of the relations between these two notions of context, see Stalnaker [2014], Rothschild and Yalcin [2015].
} 
truth-conditional approaches to theorizing about meaning are not thereby prevented from talking about the CCPs of sentences. What such theorists want to reject is not the idea that sentences have CCPs, but rather the idea that the compositional semantic value of a sentence is identical to its CCP. This is the idea of compositional dynamicness:

Compositional dynamicness. The compositional semantic values of sentences are context-change potentials - functions which map a conversational state to a new conversational state.

Something like this idea is perhaps what most theorists have in mind when they think of dynamic semantics.

At first glance, this is a simple and straightforward idea. On this understanding of 'dynamic', dynamic semantics is meant to be a clear alternative to a more ordinary, static truth-conditional compositional semantics in the style of, say, Lewis [1970] or Montague [1973]. The question whether to go dynamic in this sense is the question whether the most elegant and explanatory semanticspragmatics for the language fragment in question identifies the semantic values of sentences with their context-change potentials. That would seem to be a substantive question.

It helps to see what compositional dynamicness is supposed to contrast with. Theorists who reject compositional dynamicness will typically hold that the mapping from sentences to their context-change potentials is not directly given in the semantics, but rather arises out of the interaction of a non-dynamic semantics with separate pragmatic principles. The picture of the interaction between semantics and pragmatics described by Stalnaker [1978] is perhaps the paradigm example of an approach like this. Here, the compositional semantic value of a (declarative) sentence $\phi$ is something truth-conditional (in particular, it is a two-dimensional intension, broadly in the style of Kaplan [1977/1989]). This object, relative to a context (centered world), determines a proposition, the latter modeled as a set of possible worlds. The compositional semantics of $\phi$ alone thus does not get us all the way to its CCP. To get to the CCP of $\phi$, we need to make reference to a further ingredient outside of the compositional semantics proper. This is where Stalnaker introduces a pragmatic rule - the assertion rule. The assertion rule is meant to capture what Stalnaker takes to be a pragmatic convention of speech, namely the convention that when one utters a sentence $\phi$ that expresses a proposition $p$, this is normally mutually taken as a kind of "proposal" to change the conversational state by adding the information that $p$ to it. Since, for Stalnaker, conversational states are sets of possible worlds (context sets), he cashes this out formally as a proposal to intersect $p$ with the context set (as it stands after the utterance is produced). 
We have, then, two big-picture ways of associating sentences with contextchange potentials. One approach embraces compositional dynamicness, and effects this mapping directly within the compositional semantics. The other approach rejects compositional dynamicness, and instead associates sentences with their CCPs indirectly, through the interplay of a static semantics and one or more pragmatic bridge principles. The bridge principles span the gap between a sentence's static meaning and its CCP.

The point of debate between these two big pictures seems substantive. But capturing an interesting notion of compositional dynamicness is less straightforward than it may seem. We can bring this out by considering two systems for interpreting an artificial propositional language $L$ that contains only negation and conjunction (cf. van Benthem 1996, 17-18). One will be compositionally dynamic, one not. Both systems will employ the same models and the same notion of a conversational state:

Defs. A model $\mathcal{M}$ for $L$ is a pair $\langle W, I\rangle$ where $W$ is a set of possible worlds, and $I$ is an interpretation function mapping the propositional letters of $L$ to sets of worlds.

Def. A conversational state in $\mathcal{M}$ is any subset of $W_{\mathcal{M}}$.

The first system recursively associates sentences with meanings which are CCPs:

\section{System 1}

Def. For any $\mathcal{M}$, an update function $\cdot[\cdot]$ (for $\mathcal{M})$ is a function from wffs of $L$ to functions from conversational states (in $\mathcal{M}$ ) to conversational states (in $\mathcal{M}$ ) defined as follows, where $\alpha$ is any propositional letter, $\phi$ and $\psi$ are any wffs, and $c$ is any context set in $\mathcal{M}:^{5}$

$$
\begin{aligned}
& c[\alpha]=c \cap \mathcal{I}(\alpha) \\
& c[\neg \phi]=c-c[\phi] \\
& c[\phi \wedge \psi]=c[\phi][\psi]
\end{aligned}
$$

Def. $\phi$ is true at $w$ iff $w \in W[\phi]$

Def. $\psi$ is a consequence of a set of sentences $\Gamma$ iff at any world $w$ where all the sentences in $\Gamma$ are true, $\psi$ is true.

Here, the compositional semantic value of any sentence $\phi$ is a CCP, viz., $[\phi]$. So officially we have compositional dynamicness. We want to compare this system to the following non-dynamic system:

\footnotetext{
${ }^{5}$ We use postfix notation when formalizing CCPs.
} 


\section{System 2}

Def. For any $\mathcal{M}$, a static semantic value function $\llbracket \cdot \rrbracket($ for $\mathcal{M}$ ) is a function from wffs of $L$ to subsets of $W_{\mathcal{M}}$ defined as follows, where $\alpha$ is any propositional letter, $\phi$ and $\psi$ are any wffs, and $c$ is any context set in $\mathcal{M}$ :

$$
\begin{aligned}
& \llbracket \alpha \rrbracket=\mathcal{I}(\alpha) \\
& \llbracket \neg \phi \rrbracket=W-\llbracket \phi \rrbracket \\
& \llbracket \phi \wedge \psi \rrbracket=\llbracket \phi \rrbracket \cap \llbracket \psi \rrbracket
\end{aligned}
$$

Def. $\phi$ is true at $w$ iff $w \in \llbracket \phi \rrbracket$

Def. $\psi$ is a consequence of a set of sentences $\Gamma$ iff at any world $w$ where all the sentences in $\Gamma$ are true, $\psi$ is true.

This second system adopts a semantic value function mapping sentences to propositions (sets of worlds) along ordinary static lines. We assume this system comes packaged with a straightforward pragmatic rule of assertion, in the style of Stalnaker:

Assertion rule. For all $\phi$, the $\mathrm{CCP}$ of $\phi,[\phi]$, is defined as follows: for all conversational states $c, c[\phi]:=c \cap \llbracket \phi \rrbracket$.

System 2 is obviously not compositionally dynamic. The thing to notice is that despite this, it associates all sentences with just the same CCPs as System 1. In a sense, System 2 supplies a means of writing compositional dynamicness out of the story altogether, compatible with preserving all the CCPs associated with sentences by System 1 .

When we compare System 1 and System 2, it becomes tempting to say that compositional dynamicness was not actually present in any deep sense in System 1 in the first place (cf. von Fintel and Gillies [2007]). It becomes tempting to say that System 1 is not "truly dynamic" (Groenendijk and Stokhof [1991a]). After all, the possibility of a straightforward static reformulation undermines the idea that dynamicness at the compositional level was really necessary.

The challenge, of course, is to articulate what exactly the deeper sense of compositional dynamicness would be. If the fact that a compositional semantics takes a dynamic shape (i.e., that it is a compositional assignment of CCPs to sentences) does not settle whether it is "truly dynamic", what does?

Here is one line of approach to this question that we will not take. An intuitive idea one encounters in the literature is the idea that dynamic semantics is distinguished by the essential role that local contexts play in the compositional semantic process (see Schlenker [2009], Dever [2013] for two recent discussions). 
It very well may be that there is an attractive characterization of what a "truly dynamic" compositional semantics amounts to in terms of the concept of a local context. But we have no suggestions to offer in that vein here. Flagging this idea as a potential line of inquiry for future work, we set it aside.

So the question remains: what (if anything) makes for a "truly dynamic" compositional semantics?

\section{Conversation systems dynamicness}

We want to leave the question hanging for the moment. We turn now to our second kind of dynamicness. Once we spell this notion out, we will have some new resources for thinking about what interesting compositional dynamicness could amount to.

The second notion of dynamicness applies to a language system in abstraction from its compositional semantics. It has to do only with the characteristic forms of conversational state update that the language system allows. The relevant level of abstraction is what Rothschild and Yalcin [2015] call the conversation system level of description:

Def. A conversation system is a triple $\langle L, C, \cdot[\cdot]\rangle$, where $L$ is a set of sentences, $C$ is a set of conversational states, and $\cdot[\cdot]$ is an update function from $L$ to a set of CCPs on $C$ (i.e., . [.] $: L \rightarrow(C \rightarrow C)$ ).

System 1 and System 2 differ in the compositional semantic values that they associate with sentences. But they are indistinguishable at the conversation systems level of abstraction: they each determine exactly the same conversation system. This level of description enables us to capture, in a precise way, what the two systems have in common.

We can use the conversation system level of description to theorize more precisely about some vague ideas that are often associated with debates about static versus dynamic approaches to linguistic meaning and use. In particular, we can take a step towards formalizing the following two ideas that are often associated with dynamic approaches, and which are sometimes thought to recommend them. The first idea is some sentences have CCPs whose effect is not, or not merely, to add a proposition to the information captured by the conversational state. The second idea is that the way that the CCP of a sentence updates a conversational state may depend on features of the input conversational state: the effect of a CCP can be sensitive to the history of the discourse, as the latter is reflected in the conversational state. In a nutshell, here are two ideas dynamic approaches characteristically want to reject: 
Propositionality. Conversational update is always just a matter of adding a proposition to the conversational state.

InsEnsitivity. Conversational update is always insensitive to the input conversational state.

These are intuitive ideas. Intuitive ideas can rarely be perfectly formalized. Still, we can imperfectly theorize about them, using the notion of a conversational state. We could employ formal counterparts of these ideas to isolate some precise senses of "static" and "dynamic" at the conversation systems level of description. This is a primary objective of Rothschild and Yalcin [2015]. (Much of the discussion in this section will draw from this paper.)

One possible notion of an austerely static conversation system is the idea of a system satisfying both PROPOSITIONALITY and INSENSITIVITY. Systems 1 and 2 above are examples of systems that satisfy these two properties. Every CCP is equivalent to an operation which adds a proposition to the input conversational state; and the proposition which gets added is never sensitive to what the input conversational state is.

We should like to formalize, insofar as possible, what it is to be an austerely static conversation system in this sense. As a first step, we can observe that the conversation system determined by Systems 1 and 2 is intersective:

Def. A conversation system $\langle L, C, \cdot[\cdot]\rangle$ is intersective just in case $C \subseteq \mathcal{P}(W)$ for some set $W$, and there exists some set $P \subseteq \mathcal{P}(W)$ such that for all $\phi \in L$, there exists $p \in P$ such that for any $c \in C$, $c[\phi]=c \cap p$.

The basic notion of an intersective conversation system has often been in the air in discussions of what makes for the distinction between a static and a dynamic approach to meaning and use (though not under that particular label). Indeed, the technical result which is most often cited in connection with the static/dynamic divide is a result about intersectivity, due to van Benthem. This result isolates a pair of properties as jointly characteric of the intersective conversation systems:

Fact 1 (van Benthem [1986]). A conversation system $\langle L, C, \cdot[\cdot]\rangle$ is intersective just in case for all $\phi \in L$ and $c \in C$ :

(i) $c[\phi] \subseteq c \quad$ (eliminativity)

(ii) $c[\phi]=\bigcup_{w \in c}\{w\}[\phi] \quad$ (distributivity) 
The failure of one or both of these properties is often claimed to be a key hallmark of dynamicness (Groenendijk and Stokhof [1991a], van Benthem [1996], von Fintel and Gillies [2007], Muskens et al. [2011]).

What is not always acknowledged in discussions of this result, however, is that it occurs at the conversation systems level of description. Again, this level of description abstracts from the details of the compositional semantics of the language in question. If eliminativity or distributivity fails in the conversation system for a language fragment, it is not obvious what consequence this has, if any, for the semantics of the language - in particular, for the question whether it is compositionally dynamic. This is not to say that van Benthem's result is not interesting; on the contrary, we think it obviously is interesting. It is just important to keep in mind that its interest occurs at a specific level of abstraction, and its bearing on the architecture of semantic theory far from obvious.

Remaining at the conversation systems level, Rothschild and Yalcin [2015] offer to capture the concept of staticness fixed upon by van Benthem with a formal notion intimately related to, but more general than, that of intersectivity. Here is the definition:

Def. A conversation system $\langle L, C, \cdot[\cdot]\rangle$ is strongly static just in case there exists an intersective conversation system $\left\langle L, C^{\prime}, \cdot[\cdot]^{\prime}\right\rangle$ and a bijection $f$ from $C$ to $C^{\prime}$ such that $f(c)[\phi]^{\prime}=f(c[\phi])$, for all $\phi \in L$ and $c \in C$.

This technical notion of staticness is more neutral about the nature of conversational states than the notion of intersectivity. (The latter notion but not the former applies only when conversational states are sets, for example.) It captures a broader variety of the possible conversation systems that manifest both PROPOSITIONALITY and INSENSITIVITY.

Just as van Benthem provided characterizing properties for the class of intersective conversation systems, we can provide characterizing properties for the class of conversation systems which are static in the above sense. This takes us to a second result:

Fact 2 (Rothschild and Yalcin [2015]). A conversation system $\langle L, C, \cdot[\cdot]\rangle$ is strongly static just in case for all $\phi, \psi \in L$ and $c \in C$ :

(i) $c[\phi]=c[\phi][\phi] \quad$ (idempotence)

(ii) $c[\phi][\psi]=c[\psi][\phi] \quad$ (commutativity)

Given Fact 2, one quite natural idea of dynamicness at the conversation systems level is simply the idea of a conversation system that violates either idempo- 
tence or commutativity. This formal result sits naturally with much existing discussion of what makes for dynamicness. The violation of one or both of these properties - especially, the violation of commutativity — has often been associated with the motivation for dynamic approaches to semantics (for relevant discussion, see Groenendijk and Stokhof [1989], Groenendijk et al. [1996], van Eijck and Visser [2012], Lewis [2014]). Dynamic semantic systems are usually explicitly designed to exhibit a sensitivity to the order in which the sentences of a discourse occur. For order to matter is for commutativity to fail.

But again, dynamicness in the present sense-non-strong-staticness of the conversation system - does not per se imply compositional dynamicness (as Rothschild and Yalcin [2015] emphasize). It would be a nonsequitur to move from the fact that the conversation system for a language fragment is non-static (in the technical sense of 'static' just defined) to the conclusion that the language fragment must have a compositionally dynamic semantics. This is one of the main reasons it is important to separate these two notions of dynamicness.

Continuing the search for formally interesting classes of conversation systems, one might seek a characterization of the class of conversation systems which accept PROPOSITIONALITY, but which do not necessarily accept INSENSITIVITY. Any approach to natural language meaning and update which fits the stereotype of a static approach, and which construes conversational state update as proposition-adding, will certainly allow that the proposition expressed by a sentence can be a function of context; and one important feature of the context is, of course, the state of the conversation. If the proposition that $[\phi]$ adds to an input conversational state $c$ can vary as a function of $c$, then INSENSITIVITY fails, and the associated conversation system is not static in the above technical sense. All the same, in our experience, many theorists have the feeling that such systems should be deemed 'static', if only in a less austere sense of 'static' than the one just defined above. This feeling presumably owes to the fact that it is easy to see how a context-sensitive (especially: a conversational state-sensitive) truth-conditional compositional semantics could, together with a simple Stalnakerian rule of assertion, determine a conversation system which violates INSENSITIVITY. There are many examples in the literature with this shape; see Rothschild and Yalcin [2015]. ${ }^{6}$

Here again, however, we should be careful about levels of abstraction. If a conversation system that is not strongly static in the above technical sense can nevertheless be understood as determined, in part, by a context-sensitive truth-

\footnotetext{
${ }^{6}$ Indeed, it can be tempting to think that any kind of context-sensitivity, including the sort of context-sensitivity familiar from Kaplan-like analyses of indexicals, will lead to violations of insensitivity. But the issue here is subtle. Arguably, many textbook forms of indexicality can be squared with strong staticness. See Rothschild and Yalcin [2015] for a detailed discussion.
} 
conditional semantics of some sort, then we can simply say that the semanticspragmatics of the language fragment in question is compositionally static, but non-static at the conversation systems level. These notions of dynamicness just are separable. There should not be a general expectation to the effect that any interesting notion of dynamicness at the conversation systems level should imply compositional dynamicness.

(This seems like a convenient place to note that we don't particularly care about the terminology, as long as the underlying distinctions are acknowledged. If some theorists would prefer to reserve the word 'static' for the class of conversation systems that obey PROPOSITIONALITY, leaving INSENSITIVITY out of it, that is fine with us. They will need some other name for the class of language fragments that obey PROPOSITIONALITY and InSENSITIVITY. We take it as obvious that it is an interesting and notable fact when a fragment of language has both of these properties. The availability of a very tidy characterization of this class as the commutative, idempotent systems by itself suggests we have here a natural category of conversation systems. ${ }^{7}$ We elect to call this class 'strongly static'.)

Since, as just noted, there exist stereotypically static approaches to meaning and communication which violate INSENSITIVITY, it is of interest to characterize also the class of conversation systems construable as obeying PROPOSITIONALITY but not necessarily InSENSITIVITY. This class of systems is intimately related to the class of systems that are eliminative in the sense of the result of van Benthem's noted above. We can define this class as follows:

Def. A conversation system $\langle L, C, \cdot[\cdot]\rangle$ is eliminative just in case $C \subseteq \mathcal{P}(W)$ for some set $W$, and there exists some set $P \subseteq \mathcal{P}(W)$ such that for all $\phi \in L$ and any $c \in C$, there exists $p \in P$ such that $c[\phi]=c \cap p$.

We put the definition in this way to bring out how it differs from the definition of intersective systems given above: the only difference is that we swap the final two quantifiers. An equivalent but simpler definition of 'eliminative' would be:

Def. A conversation system $\langle L, C, \cdot[\cdot]\rangle$ is eliminative just in case $C \subseteq \mathcal{P}(W)$ for some set $W$, and for all $\phi \in L$ and any $c \in C, c[\phi] \subseteq c$.

\footnotetext{
${ }^{7}$ The point that the notion of a conversation system applies to fragments of language, and not necessarily to entire languages, is important. We doubt that anyone thinks that the conversation system appropriate for some entire natural language is static in the technical sense we have defined. Surely any natural language supplies mechanisms for varieties of conversational update that are not strongly static. Questions of staticness have real interest only for targeted fragments of language.
} 
Eliminativity captures the idea of PROPOSITIONALITY when, for example, conversational states and propositions are both modeled in terms of sets of possible worlds (as, for example, in Stalnaker [1978]). If propositions are sets of worlds and conversational states are sets of worlds, then update by proposition-adding amounts to intersecting the proposition in question with the prior conversational state. If all update is proposition-adding, then update is the sort of thing that can change the conversational state only by eliminating possibilities.

If we are seeking a natural abstract characterization of the notion of PROPOSiTIONALITY, we do well not to stop at the class of eliminative systems. The eliminativity property only applies when we assume that conversational states are objects we can model as sets. But there is no particular reason to think that the intuitive notion of PROPOSITIONALITY requires the assumption that conversational states must be modeled in this way. As before, we can arrive at a more general underlying property by considering the class of systems that are isomorphic to the eliminative systems. Rothschild and Yalcin [2015] call this class of systems weakly static:

Def. A conversation system $\langle L, C, \cdot[\cdot]\rangle$ is weakly static just in case there exists an eliminative conversation system $\left\langle L, C^{\prime}, \cdot[\cdot]^{\prime}\right\rangle$ and a bijection $f$ from $C$ to $C^{\prime}$ such that $f(c)[\phi]^{\prime}=f(c[\phi])$, for all $\phi \in L$ and $c \in C$.

The class of weakly static conversation systems corresponds to one reasonable attempt to formally cash out the idea of PROPOSITIONALITY.

We can ask, as we did with the static conversation systems, whether this class of systems can be independently characterized with one or more natural formal properties. Rothschild and Yalcin [2015] show that the class of weakly static systems corresponds to the class of systems that are antisymmetric, where:

Def. A conversation system $\langle L, C, \cdot[\cdot]\rangle$ is antisymmetric just in case, for all $c, c^{\prime} \in C$ : if $c$ is reachable from $c^{\prime}$ by some sequence of updates, and $c^{\prime}$ is reachable by $c$ by some sequence of updates, then $c=c^{\prime} .8$

To state it explicitly:

Fact 3 (Rothschild and Yalcin [2015]). A conversation system is weakly static just in case it is antisymmetric.

\footnotetext{
${ }^{8}$ The relevant notion of "reachable" is the obvious one: $c^{\prime}$ is reachable from $c$ in a conversation system $\langle L, C, \cdot[\cdot]\rangle$ just in case there exists a sequence $\left[\phi_{1}\right], \ldots,\left[\phi_{n}\right]$, with $\phi_{1}, \ldots, \phi_{n} \in L$, such that $c\left[\phi_{1}\right], \ldots,\left[\phi_{n}\right]=c^{\prime}$.
} 
The antisymmetric systems are the system where "there is no going back". This does seem to capture what is perhaps the core formal feature of PROPOSITIONALITY. If you can at most add information to the conversational state, then no update is such that it will take you to a state that you have already passed. Moving back to such a state would require losing information. When update can only be proposition-adding, update can never result in information loss.

The class of weakly static systems provides another interesting boundary at the conversational systems level of description. ${ }^{9}$ When we transgress this boundary, we are not in a place where updates are all construable as propositionadding; and that is idea that many would associate with the language's having, in some interesting sense, a dynamic character.

At the beginning of the paper, we described conversation systems dynamicness like this:

Conversation systems dynamicness. The context-change potentials of the sentences of the language fragment are not each equivalent to an operation which adds a proposition to the background information of the conversation.

We are now in position to see why this was a rough first approximation. In light of the various formal distinctions we have drawn in this section, and in particular Facts 2 and 3, we can replace this with the following more refined terminology:

Weak conversation systems dynamicness. The conversation system of the language fragment is not both commutative and idempotent.

Strong conversation systems dynamicness. The conversation system of the language fragment is not antisymmetric.

Thus conversation systems dynamicness breaks down into two further subnotions. Weak conversation systems dynamicness is compatible with the possibility that PROPOSITIONALITY is still true; what it is in tension with is the possibility that PRopositionality and Insensitivity are both true. Strong conversation systems dynamicness, by contrast, is in a basic tension with PROPOSITIONALITY.

Our claim certainly isn't that our ways of formalizing the intuitive notions of PROPOSITIONALITY and INSENSITIVITY at the conversation systems level are the only possible ways of doing so. We would be pleased if our discussion stimulated further inquiry into other possible formalizations.

\footnotetext{
${ }^{9}$ See Rothschild and Yalcin [2015] for some further discussion of some other distinctions that can be made at this level of description. See also Valby [2015] for additional results.
} 


\section{Compositional dynamicness again}

The conversation systems level of description might seem to provide a new angle from which to get more of a grip on the idea of compositional dynamicness. Given a conversation system for some language $L$, we can ask: is there a compositional mapping from any sentence $\phi$ in $L$ to its CCP $[\phi]$ in the conversation system? In other words, can the conversation system be recovered from some compositional dynamic semantics? Here what we are asking is whether the contribution that $\phi$ makes to the determination of the CCPs of complex expressions in which it occurs is completely captured by $[\phi]$. When this is true of a conversation system $\langle L, C, \cdot[\cdot]\rangle$, the following holds:

Substitution of update equivalents. For all sentences $\alpha, \beta, \phi \in L$, if $[\alpha]=[\beta]$, then for any $\phi$, if $\phi$ is a sentence with one or more occurrences of $\alpha$, and $\phi^{\alpha / \beta}$ is the result of replacing every occurrence of $\alpha$ in $\phi$ with $\beta$, then $[\phi]=\left[\phi^{\alpha / \beta}\right]$.

We can lay this down as a requirement on one legitimate notion of compositional dynamicness:

Substitutability. A language $L$ admits of a compositional dynamic semantics relative to a conversation system for $L$ only if the conversation system satisfies Substitution of update equivalents.

We will take this to be a necessary, not sufficient, condition for when a language admits of a compositional dynamic semantics.

If we looked only at examples like System 1 and System 2, we might get the impression that if we have a system equipped with a static compositional semantics, then it is always a routine matter to turn it into one with dynamic semantic values instead. This is not so. It is a substantive fact when a conversation system can be seen as induced by a compositional dynamic semantics. To see this, and to get a sense of the content of Substitutability, we can describe a system with the following properties: (i) it has a static compositional semantics; (ii) it has a dynamic conversation system; and (iii) its conversation system cannot be seen as induced by a dynamic compositional semantics for the

language. Consider what we will call System 3. System 3 assumes the same language and compositional semantics as System 2, but it adopts a very different pragmatic update rule. Instead of the Assertion rule, System 3 employs the following bizarre update rule:

Strange rule. For all conversational states $c$ and sentences $\phi$, 


$$
c[\phi]:=\left\{\begin{array}{l}
p \text { if } \llbracket \phi \rrbracket=q ; \text { otherwise } \\
\llbracket \phi \rrbracket
\end{array}\right.
$$

(where $p, q$ are some particular sets of worlds such that $p \neq q$.)

For notational convenience, we denote a wff and the proposition it expresses using the same symbol, with context disambiguating; thus $p=\llbracket p \rrbracket, q=\llbracket q \rrbracket$, and so on. System 3 is, of course, not a plausible candidate for any fragment of natural language, but it is useful for illustrating a conceptual point. In particular, notice that in System 3, $[p]=[q]$, but $[\neg p] \neq[\neg q]$. (By the definitions, for any $c, c[\neg p]$ is just the proposition $\neg p$, and $c[\neg q]$ is just the proposition $\neg q$.) Accordingly, there is no function which maps $[p]$ to $[\neg p]$ and $[q]$ to $[\neg q]$. A fortiori there is no way of thinking of negation as expressing such a function. There is no way to construe the negation symbol in this system as expressing a function which takes as its sole argument the CCP of the sentence which is its scope, and which returns as output the $\mathrm{CCP}$ of the whole negated sentence. This is one relatively clear sense in which compositional dynamicness can be said to fail.

System 3 is strongly dynamic in the conversation systems sense defined at the end of last section, because it is clearly not antisymmetric. Nevertheless, if compositional dynamicness is understood to require Substitutability, then System 3 does not admit of a compositional dynamic semantics. It is natural to regard the compositional semantics of the language is static. Interesting dynamics appears at the conversation systems level, owing to the strange update rule. The conversational dynamics generated by this rule cannot be moved into the compositional semantics, as the context change triggered by $q$ cannot be identified with the compositional semantic contribution of $q$.

So it is a nontrivial fact when a conversation system can be matched with a dynamic semantics that generates it. Let us say that if a semantics is compositional and is such that for all $\phi, \llbracket \phi \rrbracket$ equals $[\phi]$ as the latter is defined in some conversation system $C$, then this semantics is at least surface dynamic relative to $C$ :

Def. A compositional semantic value function $\llbracket \cdot \rrbracket$ for a set of expressions $E$ is surface dynamic relative to a conversation system $\langle L, C, \cdot[\cdot]\rangle$, where $L \subseteq E$, just in case it is compositional and $\llbracket \phi \rrbracket=[\phi]$ for any $\phi \in L .^{10}$

\footnotetext{
${ }^{10} \mathrm{As}$ is familiar, there are various technical notions of "compositional". We are happy to leave this definition as a definition-schema which can be precisified using various notions of compositionality - though we of course assume that any compositional dynamic semantics satisfies Substitutability.
} 
System 1 is surface dynamic, whereas Systems 2 and 3 are not. We can also define a notion that separates Systems 2 and 3:

Def. A conversation system is dynamically composable if there exists some compositional semantics which is surface dynamic relative to it.

The conversation system of System 2 is dynamically composable (as System 1 illustrates) whereas the conversation system of System 3 is not.

Now let us pick up the thread of our earlier discussion of System 1 and 2. Recall we discussed the intuition, expressed in various places in the literature, that System 1 is only superficially dynamic. Granting that the semantics of System 1 is surface dynamic, is there some nontrivial sense in which it is merely surface dynamic? Can we frame some further notion(s) of "compositional dynamicness" relative to which this semantics is not "deeply" dynamic?

One natural thought here would be to leverage the two notions of dynamicness we have already framed at the conversation systems level. We could try saying that System 1 is dynamic, but avoidably so in a technical sense:

Def. A compositional semantics is avoidably dynamic relative to conversation system $C$ iff it surface dynamic relative to $C$ and $C$ is strongly static.

Since any strongly static conversation system can be generated by a static compositional semantics for the language plus an Assertion-like update rule - we know this because in such systems a one-one correspondence exists between $[\phi]$ and static values $\llbracket \phi \rrbracket$-we capture one sense in which it could be said that the compositional semantics of System 1 is not "truly dynamic": we didn't need a (surface) dynamic semantics to generate the corresponding conversation system.

Proceeding in this fashion, we can give matching definitions for when a compositional semantics is (weakly or strongly) dynamic:

Def. A compositional semantics is weakly dynamic relative to conversation system $C$ iff it surface dynamic relative to $C$ and $C$ is not strongly static.

Def. A compositional semantics is strongly dynamic relative to conversation system $C$ iff it surface dynamic relative to $C$ and $C$ is not weakly static.

On this approach, the dynamicness of a (surface dynamic) compositional semantics is dictated by the dynamicness of the conversation system it gives rise to. 
These definitions of compositional dynamicness are fine as far as they go. We bring them up in order to separate them from other notions, and in order to note that they don't really go very far. If a compositional semantics is (weakly or strongly) dynamic as just defined, does it follow that its conversation system could not also be generated by some kind of package of a static semantics plus one or more pragmatic bridge principles? No, this does not follow. In System 3 , we have already seen an illustration of the way that a static semantics might easily give rise to very nontrivial dynamics at the conversation systems level. It is not hard to dream up systems that fit the stereotype of a static system, and yet give rise to nontrivial conversation systems dynamicness.

Indeed, one does not have to dream up such systems; one can find them in the literature. Consider for example the following system, which most would regard as an example of a static system. The system involves a trivalent static semantics, and the matching pragmatic assertion rule reflects one standard idea about how to deploy a trivalent semantics to model presupposition. ${ }^{11}$

\section{System 4}

Def. A trivalent model $\mathcal{M}$ for $L$ is a pair $\langle W, I\rangle$ where $I$ is an interpretation function mapping propositional letters of $L$ to functions from $W$ into $\{0,1, \#\}$.

Def. A trivalent valuation function $\llbracket \cdot \rrbracket_{\mathcal{M}}$ is a compositional mapping from $L$ to functions from from $W_{\mathcal{M}}$ into $\{0,1, \#\}$. (As for how the connectives are defined, it doesn't really matter for the purposes of the present point; the strong or weak Kleene semantics for connectives may be assumed, for instance.)

Def. $\phi$ presupposes $\psi$ iff for any $w$, if $\llbracket\left[\psi \rrbracket^{w} \neq 1 \text {, then } \llbracket \phi\right]^{w}=\#$

Def. A conversational state for $\mathcal{M}$ is any subset of $W_{\mathcal{M}}$ or \# (the error state).

Trivalent assertion rule. For all conversational states $c$ in $\mathcal{M}$ and sentences $\phi$,

$$
c[\phi]:=\left\{\begin{array}{l}
\left.c \cap\{w: \llbracket[\phi]]^{w}=1\right\} \text { if } c \neq \# \text { and there is no } w \in c \text { s.t. }\left[[\phi]^{w}=\#\right. \\
\# \text { otherwise }
\end{array}\right.
$$

Such systems are clearly eliminative. Now when $\phi$ presupposes $\psi$, systems like this will allow for cases where $c[\psi][\phi] \neq \#$ whereas $c[\phi][\psi]=\#$. Thus

\footnotetext{
${ }^{11}$ The assertion rule for trivalent formulas is from Stalnaker [1973]. For discussion of the trivalent approach to presupposition see Peters [1979], Beaver and Krahmer [2001], and Fox [2008].
} 
the conversation system associated with this kind of approach is not generally commutative. This sort of system is thus generally only weakly static.

Observe further that the trivalent assertion rule is a one-to-one mapping from trivalent semantic values to CCPs. So we can be assured that the conversation system of System 4 could be generated by a weakly dynamic semantics.

What is the larger lesson here? The lesson is that if a compositional semantics is dynamic in one of the senses defined above, it does not follow that a dynamic semantics is unavoidable (i.e., that we couldn't have given a compositional static semantics combined with an assertion rule that induces the same conversation system). ${ }^{12}$ But recall that the alleged worry about System 1 was that it was not unavoidably dynamic - hence not "truly" dynamic. If that really was a worry for the idea of identifying compositional dynamicness with mere surface dynamicness, then this worry also afflicts the definitions of compositional dynamicness supplied above. These definitions do not capture the idea of a conversation system which is such that it can only be compositionally induced by a semantics which is dynamic in shape - that is, whose only matching compositional semantics is surface dynamic. (Thus our definition of "avoidably dynamic" fails to fully live up to its name.) We might call such systems (if they exist) essentially dynamic. If there were clear examples of fragments of natural language having this property, it would be a powerful form of argument for dynamic approaches to compositional semantics. But it seems to us that the relevant technical notion of "essentially dynamic" is elusive. Our aim has been to draw this out.

One overall reaction to this state of affairs is deflationary: perhaps there just is no interesting notion of compositional dynamicness going beyond surface dynamic. A second reaction is to keep looking. As noted earlier, it may be possible to define an interesting notion of compositional dynamicness in terms of the concept of a local context: the idea might be that a truly dynamic compositional semantics is one that makes essential use of local contexts. The challenge for this kind of characterization is (at least) to formally clarify the relevant notions of "essential use" and "local context".

Meanwhile, we should like to criticize one kind of motivation for seeking an interesting characterization of when compositional dynamicness is absolutely needed. It is sometimes assumed that static semantics has some sort of presumptive status, that we should resort to a dynamic semantics only if we must - if we can find no way to state a static alternative covering the same data. This viewpoint encourages the question: how do we tell when compositional dynamicness

\footnotetext{
${ }^{12}$ For another example, consider the superficially static approach to epistemic modals given in Yalcin [2007], which gives rise to basically the same kind of conversation system as that generated by the weakly dynamic semantics of Veltman [1996].
} 
is absolutely needed? While this question may or may not have an interesting answer, it is far from clear what would motivate the background viewpoint. We can discern no sense in which static approaches to meaning and communication are ceteris paribus simpler, in some way that would accord them presumptive status.

\section{Discourse dynamicness}

Even if we consider a language that is equipped with a static, truth-conditional semantics and a static conversation system - say, a system in the general shape of System 2 above - there is still room for another kind of dynamicness to emerge. This is what we call discourse dynamicness:

Discourse dynamicness. The truth-conditions associated with a discourse as a whole cannot always be understood as the result of first associating the sentential parts of the discourse with truth-conditions and then combining these truth-conditions.

Discourse dynamicness has to do with the way that conversational states are mapped to truth-conditions. The simplest possible mapping between a conversational state and its truth-conditions is the one assumed by Stalnaker (Stalnaker [1975, 1978]): on his view, conversational states just are truth-conditions; the mapping is the identity function. But most dynamic systems we find in the literature build more structure into conversational states than just truth-conditions. That means there is a gap between conversational states and their associated truth-conditions. A kind of dynamicness can emerge owing to this gap.

Discussions of (what we are calling) conversation systems usually involve some particular idea about how conversational states are supposed to be mapped to their truth-conditions. We can make this component of the story explicit. Suppose $\langle L, C, \cdot[\cdot]\rangle$ is a conversation system. Consider a function $t$ from $C$ into $\mathcal{P}(W)$, where intuitively, $t$ maps states in $C$ to their truth-conditions: $t(c)$ is the way the world is, according to conversational state $c$. Call a pair of a conversational state together with a function from its states to truth-conditions an extended conversation system. Our terminology from earlier carries over straightforwardly: an extended conversation system is strongly (weakly) static just in case it extends a strongly (weakly) static conversation system.

Any extended conversation system $\langle L, C, \cdot[\cdot], t\rangle$ induces an object we can call its truth-conditional counterpart:

Def. Given an extended conversation system $\langle L, C, \cdot[\cdot], t\rangle$, its truthconditional counterpart is the triple $\langle L, P, R\rangle$, where $P=\{t(c): c \epsilon$ 
$C$ \}, and where $R$ is a function which takes any $\phi \in L$ to a relation on $P$ such that for all $p, q \in P, R^{\phi}(p, q)$ iff for some $c, c^{\prime} \in C: t(c)=p$, $t\left(c^{\prime}\right)=q$ and $c^{\prime}=c[\phi]$.

If, given some extended conversation system, you can get from state $c$ to state $c^{\prime}$ by updating with $[\phi]$, then its truth-conditional counterpart will contain states $p$ and $q$ such that $p$ is the truth-condition for $c, q$ is the truth condition for $c^{\prime}$, and $p$ and $q$ are $R^{\phi}$-related. The truth-conditional counterpart of a conversation system is a way of capturing the system's dynamics from a purely truth-conditional point of view.

The truth-conditional counterpart of an extended conversation system is not necessarily a conversation system itself. $R^{\phi}$ is a relation, and may not be a function. Only when it is a function (for any $\phi$ in the relevant $L$ ) is it the case that the truth-conditional counterpart is itself a conversation system. If you have an extended conversation system in which there exist distinct states $c, c^{\prime}$ such that $t(c)=t\left(c^{\prime}\right)$ but $t(c[\phi]) \neq t\left(c^{\prime}[\phi]\right)$, then the truth-conditional counterpart of this system is not a conversation system itself, because there will be more than one set of truth-conditions $p$ such that $R^{\phi}(t(c), p)$. Within such a counterpart system we cannot think of $\phi$ as updating $t(c)$ to yield a unique new state; rather there is more than one state it is update-related to. This reflects the obvious fact that if the updates in your extended conversation system can be sensitive to more about a conversational state than just its truth-conditions, the update dynamics of the system won't be fully captured by its truth-conditional counterpart.

Related to this, an elementary fact is the following:

Fact 4. If an extended conversation system is static, it does not follow that its truth-conditional counterpart is a static conversation system.

This is to say that even if you have a static compositional semantics and a static conversational system, it may nevertheless be the case that you cannot construe the truth-conditions of any given conversational state as determinable in a simple additive way, that is, by associating each item of the discourse with truth-conditions, associating the starting conversational state with truthconditions, and then simply conjoining these truth-conditions.

We can illustrate by example. Consider System 4. The language is very simple: it contains only the atomic fragment At of the language of predicate logic, excluding the use of constant terms. So we have only things like Fxy, $G z$, and so on. In particular we have a stock of variables $\left\{x_{1}, x_{2} \ldots\right\}$, and for every $m \in \mathbb{N}$, a set $\left\{F_{1}^{m}, F_{2}^{m} \ldots\right\}$ of $m$-place predicates. We associate predicates with 
intensions in the style familiar from quantified modal logic. A model $\mathcal{M}$ for for the language is a tuple $\langle W, D, \mathcal{I}\rangle$ where: $W$ is a non-empty set of possible worlds; $D$ is a domain of possible individuals; $\mathcal{I}$ is an interpretation function taking a predicate $\mathcal{F}_{i}^{k}$ and world $w \in W$ to a (possibly empty) set of $k$-tuples of objects from $D$. A variable assignment $g$ maps each variable $x_{i}$ to some $g\left(x_{i}\right) \in D$.

Now choosing some particular model, the semantics maps sentences of At to sets of assignment-world pairs in the model, as follows:

$$
\llbracket \mathcal{F}_{i}^{k}\left(x_{1}, \ldots, x_{k}\right) \rrbracket=\left\{\langle g, w\rangle:\left\langle g\left(x_{1}\right), \ldots, g\left(x_{k}\right)\right\rangle \in V\left(\mathcal{F}_{i}^{k}, w\right)\right\}
$$

We assume that conversational states in System 4 are also modeled by sets of assignment-world pairs (from the same model). Let $K$ be the set of such states. We assume update works exactly in the style of the Assertion rule from System 2:

Assertion rule. For all $\phi \in \mathrm{At},[\phi]$ is defined as follows: for all conversational states $c \in H, c[\phi]:=c \cap \llbracket \phi \rrbracket$.

So the conversational state of System 4 is $\langle$ At, $K, \cdot[\cdot]\rangle$. Obviously System 4 is strongly static in the technical sense. Now consider the following mapping $t$ from conversational states in $H$ to their truth-conditions (i.e., to sets of worlds in the model $H$ is based on):

$$
t(c)=\{w: \text { there is some } g \text { such that }\langle g, w\rangle \in c\}
$$

This kind of mapping from conversational states to truth-conditions is familiar in the literature (cf. Heim [1982], Groenendijk and Stokhof [1991b]).

Now it is not hard to see that the truth-conditional counterpart of $\langle$ At, $K, \cdot[\cdot], t\rangle$ is not even a conversation system, let alone a static conversation system. We can give a concrete illustration. Suppose we consider an initial conversational state $c_{o}$ containing four assignment-world pairs:

$$
c_{0}=\left\{\left\langle g_{1}, w_{1}\right\rangle,\left\langle g_{1}, w_{2}\right\rangle,\left\langle g_{2}, w_{1}\right\rangle,\left\langle g_{2}, w_{2}\right\rangle\right\}
$$

We consider two objects $a$ and $b$ that exist in both $w_{1}$ and $w_{2}$. Relative to $w_{1}$, only $a$ is in the extension of predicates $F$ and $H$, and only $b$ is in the extension of $G$. Relative to $w_{2}$, only $a$ is in the extension of predicates $G$ and $H$, and only $b$ is in the extension of $F$. Furthermore, $g_{1}(x)=a$, and $g_{2}(x)=b$.

Now what we want to examine is the way the truth-conditions of the conversational state evolve under the impact of different discourses. In particular, let us compare $c_{0}[F x][H x]$ and $c_{0}[G x][H x]$. It helps to have a diagram: 


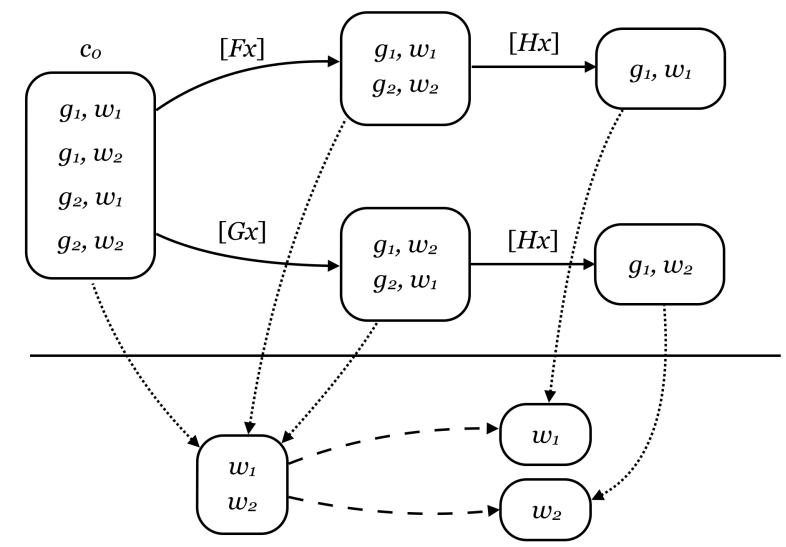

Figure 1: Discourse dynamics despite a static conversation system.

The graph above the horizontal line in Figure 1 depicts the relevant fragment of the conversation system for System 4 . The two arrows out of $c_{0}$ take you to the state as updated by $[F x]$ and by $[G x]$, respectively, and the arrows out these resulting states each take you to their respective updates on $[H x]$. The graph below the horizontal line depicts the relevant fragment of the truth-conditional counterpart for System 4. The states in this graph are truth-conditions (sets of worlds). The dotted lines from states in System 4 to truth-conditions correspond exactly to the function $t$ in the extended conversation system $\langle$ At, $K, \cdot[\cdot], t\rangle$. The dashed arrows between the truth-conditional counterpart states correspond to the relation $R^{H x}$.

Notice that the truth-conditions of the states $c_{0}, c_{0}[F x]$, and $c_{0}[G x]$ are the same: these distinct states each leave open exactly the same possible worlds. They differ only in the range of variable assignments each world left open is paired with. But that difference makes for difference in the way that these states are apt to be updated by $[H x]$; and that difference in turn affects the truthconditions of the resulting updates. The key fact here is that although $c_{0}[F x]$ and $c_{0}[G x]$ have the same truth-conditions, $c_{0}[F x][H x]$ and $c_{0}[G x][H x]$ have different truth-conditions. (The state $c_{0}[F x][H x]$ calls for there being a single 
thing that's both $F$ and $H$-only $w_{1}$ fits that bill - whereas $c_{0}[G x][H x]$ calls for there being a single thing that's both $G$ and $H$-only $w_{2}$ fits that bill.) Although the update $[\mathrm{Hx}]$ can be thought of as simply intersecting the input conversational state with a certain set of assignment-world pairs, the effect of $H x$ on the truth-conditions of an input conversational state cannot be understood in this way. The effect that $H x$ has on the truth-conditions of a conversational state depends on features of the input state other than its truth-conditions - it turns on what possible values for variables remain available for each world still left open.

The point here is trivial from a formal point of view, but it has some conceptual importance. For it highlights an important way in which the truthconditions of a discourse might fail to be a simple sum of the truth-conditions of its component parts - even if the semantics of the language is static in shape, and even the conversation system is strongly static in the technical sense (commutative and idempotent).

Dynamic views are sometimes motivated by an idea Yalcin [2012a] calls discourse primacy:

Discourse primacy. It is fundamentally entirely discourses that have truth-conditions (or more broadly, informational content). Individual sentences have truth-conditions in at best a derivative sense, insofar as they have some potential to impact the truth-conditions of a discourse.

What we have just observed is that this thesis is quite compatible with a static conception of meaning.

\section{Conclusion}

Our aim has been to tease apart notions of dynamicness at the compositional, conversation systems, and discourse levels of description. The notions of compositional dynamicness and of discourse dynamicness seem to us particularly under-explored.

A key fact that the preceding helps to clarify and bring into focus is the fact that one can in principle maintain a static compositional semantics compatible with robust conversational systems-level dynamicness and compatible with robust discourse-level dynamicness. Thus if we find linguistic evidence that suggests conversation systems-level or discourse-level dynamicness is present in some fragment of natural language, we cannot necessarily leap to the conclusion that a dynamic compositional semantics is necessary. 
On the other hand, we also don't see much reason to suppose that a dynamic semantics should be given only when it is unavoidable. First, as we have noted, it is unclear whether we can make any technical sense of the relevant notion of "unavoidable". Second, it is anyway unclear why unavoidability should be the standard - that is, why there should be some kind of presumption against a dynamic approach. If the preceding brings anything out, it is that systems based on canonically static semantics can in fact be very powerful. They do not represent some kind of austere, minimal starting point as compared to dynamic approaches. We have yet to make out any interesting sense in which a static semantics-based approach could be called the simpler hypothesis, in a way that would accord such approaches presumptive status. ${ }^{13}$ Static approaches are certainly more traditional and familiar, but whether they are simpler is a very different matter.

\section{References}

Maria Aloni. Quantification Under Conceptual Covers. PhD thesis, University of Amsterdam, 2001.

David Beaver. Presupposition and Assertion in Dynamic Semantics. Studies in Logic, Language and Information. CSLI Publications, 2001.

David Beaver. As brief as possible (but no briefer). Theoretical Linguistics, 34(3): 213-228, 2008. ISSN 0301-4428.

David Beaver and Bart Geurts. Presupposition. In Edward N. Zalta, editor, The Stanford Encyclopedia of Philosophy. Winter 2014 edition, 2014.

David Beaver and Emiel Krahmer. A partial account of presupposition projection. Journal of Logic, Language and Information, 10:147-182, 2001.

Maria Bittner. Time and modality without tenses or modals. In Renate Musan and Monika Rathert, editors, Tense across Languages, pages 147-188. Niemeyer, 2011.

Justin Bledin. Logic informed. Mind, 130(490):277-316, 2014.

Paul Dekker. Dynamic Semantics. Springer, Dordrecht, Netherlands, 2012.

Josh Dever. The revenge of the semantics-pragmatics distinction. Philosophical Perspectives, 27(1):104-144, 2013.

Kai von Fintel. Counterfactuals in a dynamic context. In Ken Kenstowicz, editor, Ken Hale: A Life in Language, pages 123-152. MIT Press, Cambridge, MA, 2001.

\footnotetext{
${ }^{13}$ Indeed, dynamic approaches operate under a constraint that static approaches don't, namely that the CCPs of sentences be compositional; thus one could imagine a case in the opposite direction, for the thesis that a dynamic approaches have presumptive status.
} 
Kai von Fintel and Anthony Gillies. An opinionated guide to epistemic modality. In Tamar Gendler and John Hawthorne, editors, Oxford Studies in Epistemology, volume 2, pages 32-62. Oxford University Press, Oxford, 2007.

Danny Fox. Two short notes on Schlenker's theory of presupposition projection. Theoretical Linguistics, 34:237-252, 2008. doi: 10.1515/THLI.2008.016.

Gerald Gazdar. Pragmatics: Implicature, Presupposition and Logical Form. Academic Press, 1979. ISBN 0122784502.

Anthony Gillies. Epistemic conditionals and conditional epistemics. Nô̂s, 38(4):585$616,2004$.

Anthony Gillies. Counterfactual scorekeeping. Linguistics and Philosophy, 30(3):329$360,2007$.

Jeroen Groenendijk. The logic of interrogation. In Tanya Matthews and Devon Strolovitch, editors, Semantics and Linguistic Theory IX, pages 109-126, 1999.

Jeroen Groenendijk and Martin Stokhof. Context and information in dynamic semantics. In Working Models of Human Perception, pages 457-486. Academic Press, 1989.

Jeroen Groenendijk and Martin Stokhof. Two theories of dynamic semantics. In Logics in AI, volume 478 of Lectures Notes in Computer Science, pages 55-64. Springer, Berlin, 1991a.

Jeroen Groenendijk and Martin Stokhof. Dynamic predicate logic. Linguistics and Philosophy, 14(1):39-100, 1991b.

Jeroen Groenendijk, Martin Stokhof, and Frank Veltman. Coreference and modality. In Shalom Lappin, editor, Handbook of Contemporary Semantic Theory, pages 179213. Blackwell, 1996.

Charles L. Hamblin. Mathematical models of dialogue. Theoria, 37(2):130-155, 1971. ISSN 1755-2567.

Irene Heim. The Semantics of Definite and Indefinite Noun Phrases. PhD thesis, University of Massachusetts, 1982.

Hans Kamp. A theory of truth and semantic representation. In Jeroen A. Groenendijk, Theo Janssen, and Martin Stokhof, editors, Formal Methods in the Study of Language, pages 277-322. Mathematisch Centrum, University of Amsterdam, Amsterdam, 1981.

David Kaplan. Demonstratives. In Joseph Almog, John Perry, and Howard Wettstein, editors, Themes from Kaplan, pages 481-563. Oxford University Press, Oxford, $1977 / 1989$. 
Lauri Karttunen. Discourse referents. In Proceedings of the 1969 Conference on Computational Linguistics, pages 1-38. Association for Computational Linguistics, 1969.

Lauri Karttunen. Presupposition and linguistic context. Theoretical Linguistics, 1 (1-3):181-194, 1974. ISSN 0301-4428.

Niko Kolodny and John MacFarlane. Ifs and oughts. Journal of Philosophy, CVII(3): 115-43, March 2010.

David K. Lewis. General semantics. Synthese, 22(1-2):18-67, 1970.

David K. Lewis. Scorekeeping in a language game. Journal of Philosophical Logic, 8 (1):339-59, 1979.

Karen Lewis. Discourse dynamics, pragmatics, and indefinites. Philosophical Studies, 158(2):313-342, 2012.

Karen Lewis. Do we need dynamic semantics? In Alexis Burgess and Brett Sherman, editors, Metasemantics: New Essays on the Foundations of Meaning, pages 231-258. Oxford University Press, 2014.

Richard Montague. The proper treatment of quantification in ordinary English. In J. Hintikka, J. Moravcsik, and P. Suppes, editors, Approaches to Natural Language: Proceedings of the 1970 Stanford Workshop on Grammar and Semantics, pages 22142. D. Reidel, 1973.

Sarah Moss. On the pragmatics of counterfactuals. Noûs, 46(3):561-586, 2012.

Sarah Murray. Varieties of update. Semantics and Pragmatics, 7(2):1-53, 2014.

Reinhard Muskens, Johan van Benthem, and Albert Visser. Dynamics. In Johan van Benthem and Alice ter Meulen, editors, Handbook of Logic and Language, pages 607-670. Elsevier, 2nd edition, 2011.

Stanley Peters. A truth-conditional formulation of Karttunen's account of presupposition. Synthese, 40:301-316, 1979.

Craige Roberts. Information structure in discourse: Towards an integrated formal theory of pragmatics. In Jae-Hak Yoon and Andreas Kathol, editors, OSU Working Papers in Linguistics, volume 49, pages 91-136. The Ohio State University Department of Linguistics, 1996.

Craige Roberts. Information structure in discourse: Towards an integrated formal theory of pragmatics. Semantics and Pragmatics, 5(6):1-69, 2012.

Daniel Rothschild. Explaining presupposition projection with dynamic semantics. Semantics and Pragmatics, 4(3):1-43, 2011.

Daniel Rothschild and Seth Yalcin. On the dynamics of conversation. Nous, Online first version(doi: 10.1111/nous.12121):1-25, 2015. 
Philippe Schlenker. Anti-dynamics: Presupposition projection without dynamic semantics. Journal of Logic, Language and Information, 16(3):325-356, 2007.

Philippe Schlenker. Be articulate: A pragmatic theory of presupposition projection. Theoretical Linguistics, 34(3):157-212, 2008.

Philippe Schlenker. Local contexts. Semantics and Pragmatics, 2(4):1-78, 2009.

Robert Stalnaker. Presuppositions. Journal of Philosophical Logic, 2(4):447-457, 1973. doi: $10.1007 /$ BF00262951.

Robert Stalnaker. Pragmatic presuppositions. In Milton K. Munitz and Peter Unger, editors, Semantics and Philosophy. New York University Press, 1974.

Robert Stalnaker. Indicative conditionals. Philosophia, 5(3):269-286, 1975.

Robert Stalnaker. Assertion. In Peter Cole, editor, Syntax and Semantics 9: Pragmatics, pages 315-332. Academic Press, 1978.

Robert Stalnaker. On the representation of context. Journal of Logic, Language, and Information, 7:3-19, 1998.

Robert Stalnaker. Common ground. Linguistics and Philosophy, 25(5-6):701-21, 2002.

Robert Stalnaker. Context. Oxford University Press, Oxford, 2014.

Lawrence Valby. Some Case Studies in Algebra Motivated by Abstract Problems of Language. PhD thesis, University of California, Berkeley, 2015.

Johan van Benthem. Essays in Logical Semantics. Studies in Linguistics and Philosophy. Reidel, Dordrecht, 1986.

Johan van Benthem. Exploring Logical Dynamics. Center for the Study of Language and Information, Stanford, CA, USA, 1996.

Jan van Eijck and Albert Visser. Dynamic semantics. In Edward N. Zalta, editor, The Stanford Encyclopedia of Philosophy. Winter 2012 edition, 2012.

Frank Veltman. Defaults in update semantics. Journal of Philosophical Logic, 25(3): 221-61, 1996.

Frank Veltman. Making counterfactual assumptions. Journal of Semantics, 22(2): 159-180, 2005.

Malte Willer. A remark on iffy oughts. Journal of Philosophy, 109(7):449-461, 2012.

Malte Willer. Dynamics of epistemic modality. Philosophical Review, 122(1):45-92, 2013a.

Malte Willer. Indicative scorekeeping. In Proceedings of the 19th Amsterdam Colloquium, pages 249-256, 2013b. 
Seth Yalcin. Epistemic modals. Mind, 116(464):983-1026, 2007.

Seth Yalcin. Dynamic semantics. In Gillian Russell and Delia Graff Fara, editors, Routledge Handbook of The Philosophy of Language, pages 253-279. Oxford University Press, 2012a.

Seth Yalcin. Context probabilism. In Maria Aloni, Vadim Kimmelman, Floris Roelofsen, Galit Weidman Sassoon, Katrin Schulz, and Matthijs Westera, editors, Proceedings of the Eighteenth Amsterdam Colloquium, volume 7218 of Lecture Notes in Computer Science, pages 12-21. Springer, 2012b.

Seth Yalcin. A counterexample to Modus Tollens. Journal of Philosophical Logic, 41 (6):1001-1024, 2012c.

Seth Yalcin. Epistemic modality de re. Ergo, 2(19):475-527, 2015. 